\title{
Humanoid Robot Head Design Based on Uncanny Valley and FACS
}

\author{
Jizheng Yan, ${ }^{1}$ Zhiliang Wang, ${ }^{2}$ and Yan Yan $^{2}$ \\ ${ }^{1}$ School of Automation and Electrical Engineering, University of Science and Technology Beijing, Beijing 100083, China \\ ${ }^{2}$ School of Computer and Communication Engineering, University of Science and Technology Beijing, Beijing 100083, China \\ Correspondence should be addressed to Jizheng Yan; happynanhu@msn.com
}

Received 30 June 2014; Revised 25 November 2014; Accepted 26 November 2014; Published 14 December 2014

Academic Editor: Shahram Payandeh

Copyright (c) 2014 Jizheng Yan et al. This is an open access article distributed under the Creative Commons Attribution License, which permits unrestricted use, distribution, and reproduction in any medium, provided the original work is properly cited.

\begin{abstract}
Emotional robots are always the focus of artificial intelligence (AI), and intelligent control of robot facial expression is a hot research topic. This paper focuses on the design of humanoid robot head, which is divided into three steps to achieve. The first step is to solve the uncanny valley about humanoid robot, to find and avoid the relationship between human being and robot; the second step is to solve the association between human face and robot head; compared with human being and robots, we analyze the similarities and differences and explore the same basis and mechanisms between robot and human analyzing the Facial Action Coding System (FACS), which guides us to achieve humanoid expressions. On the basis of the previous two steps, the third step is to construct a robot head; through a series of experiments we test the robot head, which could show some humanoid expressions; through human-robot interaction, we find people are surprised by the robot head expression and feel happy.
\end{abstract}

\section{Introduction}

Robots play an increasingly important role in our life and work, to assist and even replace the human work in more and more fields. With the development of society, the traditional robots can't meet human beings, humanoid robots [1-3] are coming, and human beings want more and more analogue. There are many famous humanoid robots, for instance, Kismet and Leonardo made from MIT in USA, ROMAN made in Germany, and WE-robot and SAYA made in Japan, which all could surprise people and give some kind of special feelings but cannot gain some place in person's awareness. This paper divided these humanoid robots into two domains. First like a beautiful flower vase to people, human beings find it is an amazing thing, and only like to see its actions, for instance, smile, anger; these robots are almost made in Japan and have shocking appearance; second like a toy to people, we find it interesting to play with this kind of robots, which can show some facial expressions, and these robots are always made in USA and Europe.

In order to avoid these cases and simplify the whole design, this paper is meant to make a humanoid robot head instead of the whole body, which not only has good appearance, but also can show some expressions. The paper is organized as follows: in Section 1 we introduce two kinds of humanoid robots. In Section 2 we introduce the concept of the uncanny valley. In Section 3 we talk about the Facial Action Coding System (FACS). In Section 4 we design the hardware and software of the robot head and build the robot head according to the previous design. Finally in Sections 5 and 6 we test the robot head and validate our conclusion.

\section{Uncanny Valley}

People always think that robot should be more and more like us, and the trend is still so, but people gradually discovered that robots appear more like human, from the appearance to voice, and even have feeling; our sense of their familiarity increases until we come to a valley, where we feel horror, as shown in Figure 1; this relation could be called the "uncanny valley" $[4,5]$. The main purpose of making kinds of robots is based on functional application; from this standpoint, the robots must perform functions similar to human laborers, but their appearance is not evaluated. If we plot industrial robots on a graph of familiarity versus appearance, they lie near the origin, so they show a little resemblance to human 


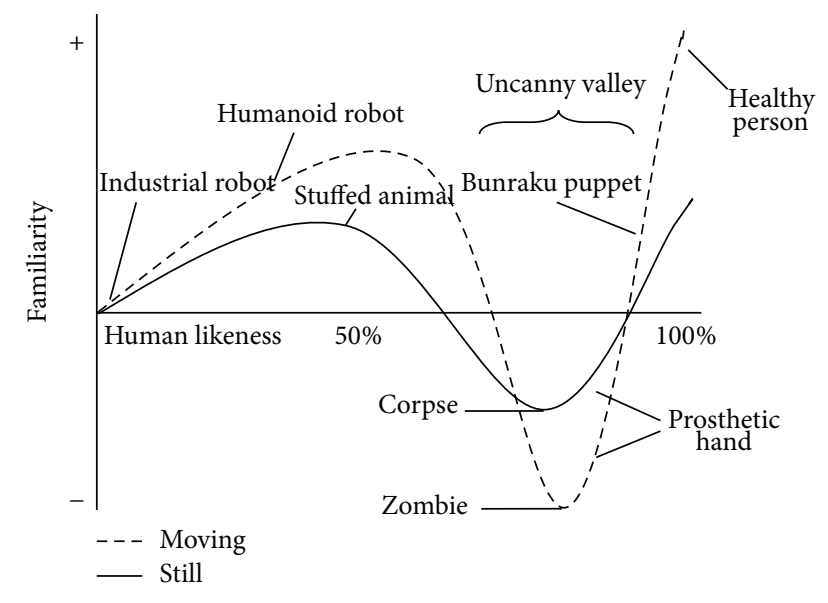

FIGURE 1: The uncanny valley.

being, and generally speaking people do not think them to be familiar. But if we go further, giving more importance to the appearance of robot than its function, robots will have a somewhat human-like appearance, for instance, a face, two arms, two legs, and a torso. This design lets children enjoy a sense of familiarity with the humanoid toy. So the toy robot is approaching the top of the first peak and a healthy person is at the top of the second peak. And when we die, we fall into the trough of the uncanny valley. Our body becomes cold, our color changes, and movement ceases. Therefore, our impression of death can be explained by the movement from the second peak to the uncanny valley as shown by the dashed line in the figure. Human beings themselves lie at the final goal of robotics, which is why we make an effort to build human-like robots. For example, robot's arms may be composed of a metal cylinder with many bolts, but to achieve a more human-like appearance we paint over the metal in skin tones. These cosmetic efforts cause a resultant increase in our sense of the robot's familiarity.

We hope to design robots or prosthetic hands that will not fall into the uncanny valley, so we have to take the first peak as the goal in building robots rather than the second. Although the second peak is higher, there is a far greater risk of falling into the uncanny valley. We predict that it is possible to produce a safe familiarity by a non-human-like design. So designers must consider this key point.

\section{Facial Action Coding System}

Facial Action Coding System (FACS) [6-9] was developed by Ekman and Friesen in 1978 to objectively measure facial activity for behavioral science investigations of the face, which provided an objective description of facial signals in terms of component motions, or "facial actions." They defined 46 action units (AUs), as shown in Table 1, and each action unit was constituted by one or several pieces muscles, to correspond to each independent motion of the face, and they combined different action units to show different facial expressions. Because units can be freely combined among the various actions, in other words, person's face
TABLE 1: Relationship between AU and Expression.

\begin{tabular}{lc}
\hline Facial expression & Action unit \\
\hline Happiness & AU6 + AU12 \\
Disgust & AU9 + AU10 \\
Sadness & AU1 + AU15 \\
Anger & AU4 + AU5 + AU24 \\
Contempt & AU10 + AU15 + AU17 \\
Surprise & AU1 + AU2 + AU5 + AU25 + AU26 \\
Fear & AU10 + AU2 + AU4 + AU5 + AU7 + AU20 \\
\hline
\end{tabular}

TABLE 2: Action unit of robot head.

\begin{tabular}{lcc}
\hline Action part & Action unit & Control point \\
\hline Eyeball & Eye turn left or right, up or down & CP3, CP14, CP15 \\
Eyelid & Upper or lower & CP1, CP2 \\
Eyebrow & Brow pull down & CP7, CP8, CP9, CP10 \\
Mandible & Open or shut mouth & CP12, CP13 \\
Mouth & Mouth oblique pull & CP5, CP6 \\
Neck & Nod or shake head & CP4, CP11 \\
\hline
\end{tabular}

may have 10,000 kinds of expression, 3000 of them maybe have the same emotional significance and people could only distinguish a few expressions. FACS continues to be the leading method for measuring facial expressions in behavioral science. According to FACS, humans share seven emotional facial expressions regardless of ethnic group, culture, and country, which are happiness, sadness, anger, fear, surprise, disgust, and contempt, so we take FACS to guide the design, to build the implementation platform. Based on FACS, we use software named FaceGen [10] to further simulate facial expressions and finally simplify these AUs.

Under the FACS, we could divide our humanoid robot head into six parts, for instance, eyeball, eyelid, eyebrow, mandible, mouth, and neck, as shown in Table 2; each control point is a servo, which could pull or push to control actions. Through FaceGen simulation, we make a decision to use 15 control points instead of 46 AUs, which also could show facial expressions and will be explained in detail in Section 5, as shown in Table 3.

\section{Design of Robot Head}

The most notable feature of artificial emotion expression robot [11-13] is to interact with the human naturally, understand human, and, at the same time, be able to face feedback. There are a number of robot systems for different purposes that are designed to interact with people; in general, these robot systems can be either embodied (the human interacts with a robot or an animated avatar) or disembodied (the human interacts through speech or text entered at a keyboard). We choose to make the embodied system of robot head, which is more real and attractive. The whole design of robot head is divided into two main parts: intelligent control system which belongs to the software work; humanoid structure and motor control system which belong to the mechanical work. Intelligent control system is the soul of 
TABLE 3: Control points of robot head.

\begin{tabular}{lc}
\hline Number & Control point \\
\hline CP1 & Left eyelid \\
CP2 & Right eyelid \\
CP3 & Turn the eye \\
CP4 & Turn the head \\
CP5 & Right mouth \\
CP6 & Left mouth \\
CP7 & Left inside eyebrow \\
CP8 & Right inside eyebrow \\
CP9 & Left outside eyebrow \\
CP10 & Right outside eyebrow \\
CP11 & Nod \\
CP12 & Left mouth \\
CP13 & Right mouth \\
CP14 & Left eye flat \\
CP15 & Right eye flat \\
\hline
\end{tabular}

the robot, which makes robot create humanoid emotion. Mechanical structure is the emotional carrier, which could change emotional information to action command.

4.1. Intelligent Control System. If a robot wants to show its own feelings in a moment, it needs to analyze not only the external environment and its own emotional state, but also signal of human language, body movements, and facial expressions and this requires modeling of the emotional state of the robot. As shown in Figures 2 and 3, we devise the control architecture of robot head, use computer vision and sensors to get information from human, and send it to three databases. After operation processing, the system could make decisions and generate homologous orders; finally we use FACS and emotional speech synthesis to show us humanoid feelings.

4.2. Mechanical Structure [14-16]. A robot with artificial emotion needs to be able to carry out a series of facial expressions and body language gestures, and facial expressions are more important and attractive to people, so the head design of humanoid expression robot is a key part. Robots obtain and analyze information, make comprehensive decisions, and finally realize their inner emotional states through the head expression, such as showing a smiling, upset, happy, or scared expression. Through taking advantage of the electronic and mechanical technology more harmony and unity, finally people can make the natural flow of emotional communication with robots.

As shown in Figure 4, the mechanical design of the robot facial expressions is based on FACS, and steering gears drive the emotional robot head; the synthesis of each feature point movement forms the robot's facial expression. For example, when people expressed surprise, the eyebrows lift and bend higher and eyebrow skin will be stretched. When eyes are wide open, upper eyelid will be pushed up, but when the chin of the face falls, mouth will open. If the head of the emotional

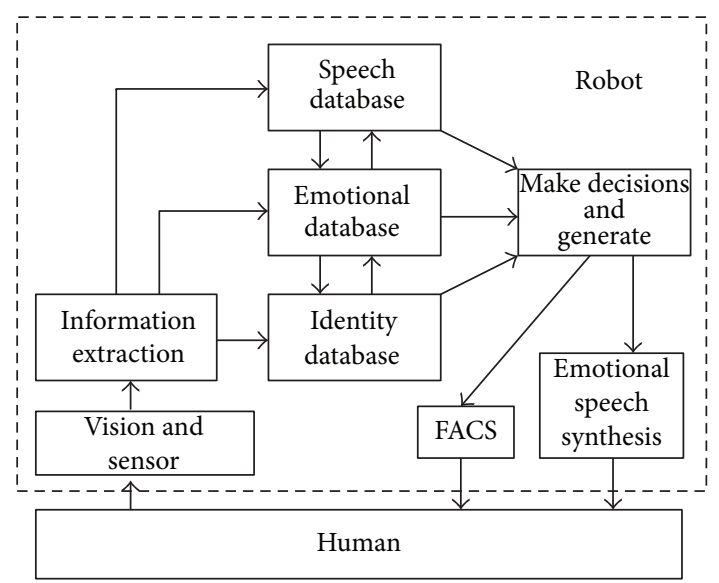

FigURE 2: Robot head control system.

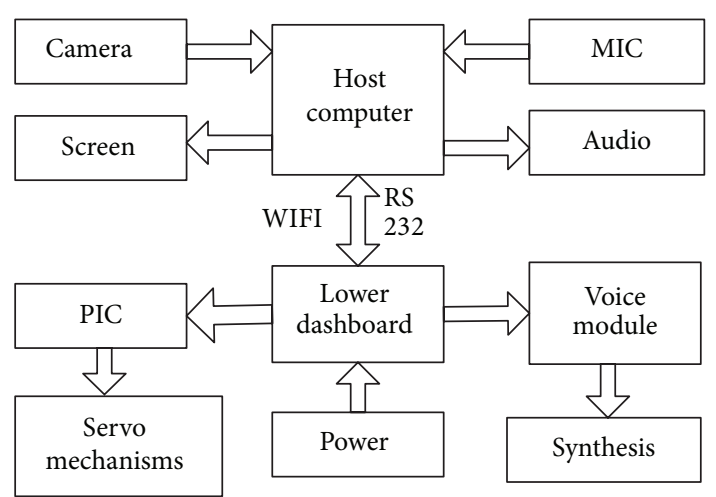

FIGURE 3: Robot motor control system.

robot design is consistent with this, you will get a surprised face. We design the whole framework of robot head, which gives us a blueprint of robot head, so we can make it step by step. From the blueprint to real, we need three steps.

Step 1. Manufacture machinery entity: we make internal structure, which has many parts, which is strict control of parts of precision; through stress analysis we further select the fairish servos, which are 15 in robot head. This step is cumbersome and needs a lot of time; it is about four weeks to us.

Step 2. Mount head shell: although we have made the "bones" of the robot head, we still cannot install facial skin directly. Human head is not only bone, but also there are a large number of muscles to fill up and we cannot simply reproduce human head, because there are a large number of servos in the robot head; we cannot use filler and have to find a way to replace muscles; through experiments we make a light-weight and rigid fiberglass shell.

Step 3. Install facial skin: we often feel bad about the head of facial damaged skin; most of the time ordinary people feel disgusted about the bare skull. I would not be surprised that you are afraid of the robot head in Figure 4. In order to make robots more like human and in order that humans seem more 

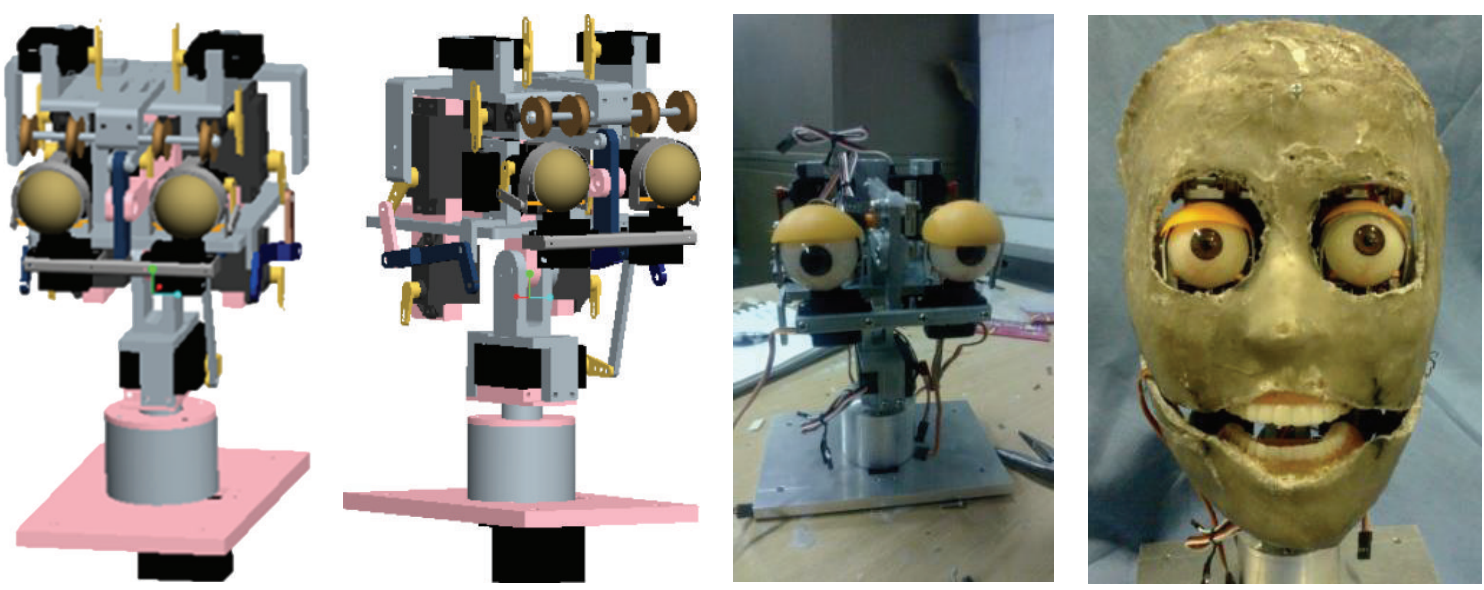

FIGURE 4: Mechanical structure of robot head.

comfortable, we install facial skin to robot using silicone and not only is this silicone similar to human skin, but the hands feel good as well.

Through the above three steps, we basically build the facial emotion expression robot head, only missing servos control, and we use PIC to control servos and voice module to process speech.

\section{Experiments}

After Section 4, we successively design the intelligent control system and build a real robot head, which could show some simple and complex facial expressions to human; in order to examine our work, this paper designs two experiments: the first is to test the uncanny valley and the second is FACS.

5.1. Experiment I. As we said in Section 2, our humanoid robot head has to avoid the uncanny valley, so we invited 100 students to evaluate the different stages of our robot head from two factors, respectively. As shown in Figure 5, first factor in red line is similarity from 1 to 10 , and 10 is the most similar. Second factor in blue line is satisfaction, which is also from 1 to 10 , and 10 is the most satisfactory; we find that the stages of 4,5 , and 6 are the uncanny valley of our robot head, and finally people are pleased with our humanoid robot.

5.2. Experiment II. In order to assess the effect of artificial expression, we invited 100 students and 50 teachers to appraise our robot head and score from 0 to 10. In order to make testers without technical bias to our robot, we chose students and teachers from other nonrelated colleges, which had not seen other physical robots, so they could only contrast our robot and human beings. Experiment is as follows.

Step 1. We let robot head show 6 basic facial expressions: pleasure, anger, fear, sadness, disgust, and neutrality and students and teachers selected the most human-like; these facial expressions formed Group 1.

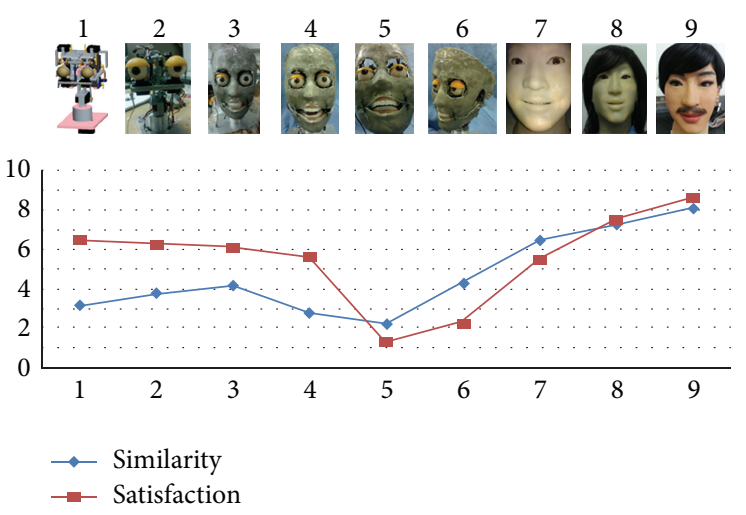

FIgURE 5: Results of our humanoid robot head.

Step 2. We let robot head show combined expression, for instance, upset and amazement, and students and teachers selected their favorite expressions, which formed Group 2.

Step 3. From Groups 1 and 2, we let students and teachers score each facial expression, and sum mean was the final score.

Step 4. From high to low of the scores we arranged Groups 1 and 2 to a new group, Group 3, and analyzed the importance of the single part in the facial expressions.

Through testers scoring and sorting, we got Group 3, as shown in Figure 6 and number 1 was the highest score.

\section{Conclusion}

From the results of human-robot interaction, we found that robot head with 15 servos could only show basic facial expressions, but that is enough for now; currently our laboratory robot is still in the research phase and we hoped to explore a path of robot emotion. Students and teachers who took the test did not feel horrible, and the most interesting part is eye. 


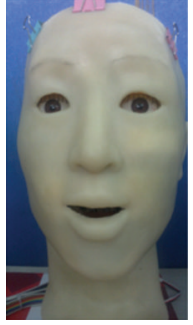

Number 1

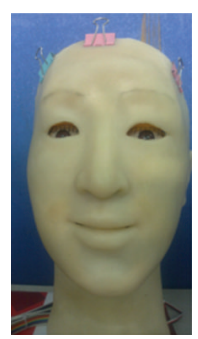

Number 2

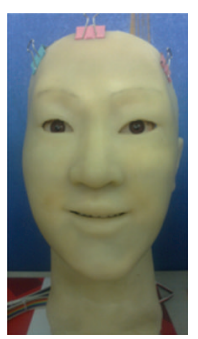

Number 3

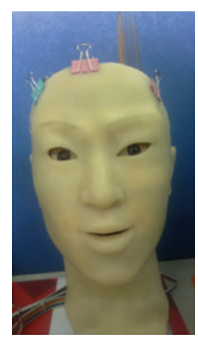

Number 4

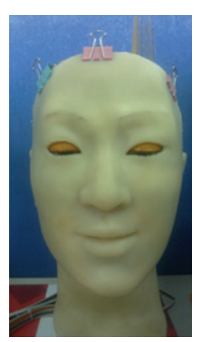

Number 5

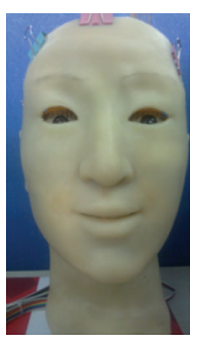

Number 6

FIGURE 6: Facial expression of Group 3.

Through analysis results we found the key of the intelligent control system and successfully avoided the uncanny valley; compared with emotional control, it is easier to make physical appearance, but not inherent control or robot emotion; most of testers thought this robot head was more than 80 percent similar to human, especially after makeup.

Under the guidance of the uncanny valley and FACS, we analyze the relevance between human and robot and by comparing the emotional foundation we find the way to achieve intelligent control of emotion and build the framework of robot. Using computer, MEMS, and related technologies, robots can have artificial emotion expressions and avoid the uncanny valley; finally we find that this robot head has the characteristics of being personalized and userfriendly and is able to achieve a harmonious interaction. But there are some shortcomings in this design: the first problem is that robots need more control points to show expressions and there is not enough space for more servos; if we want more space for servos, we have to make a bigger head, which makes it not like normal person. The next problem is that robot has lacked related equipment to collect sufficient data information, which makes the emotional decision-making extremely difficult; sometimes its decision is wrong, even ironic. Existing technology and theory cannot give the robot a lot of support, and robots do not have brain and heart to learn and think exactly like human being for now, but, with technology advancement, we think these problems will have a good solution.

\section{Conflict of Interests}

The authors declare that there is no conflict of interests regarding the publication of this paper.

\section{Acknowledgment}

This work is financially supported by the National Natural Science Foundation of China (61170117 and 61105120).

\section{References}

[1] M. A. Goodrich and A. C. Schultz, "Human-robot interaction: a survey," Foundations and Trends in Human-Computer Interaction, vol. 1, no. 3, pp. 203-275, 2007.

[2] H. Yan, M. H. Ang Jr., and A. N. Poo, "A survey on perception methods for human-robot interaction in social robots," International Journal of Social Robotics, vol. 6, no. 1, pp. 85-119, 2014.
[3] T. Nomura, "Comparison on negative attitude toward robots and related factors between Japan and the UK," in Proceedings of the 5th ACM International Conference on Collaboration Across Boundaries: Culture, Distance \& Technology (CABS '14), pp. 8790, ACM, Kyoto, Japan, August 2014.

[4] M. Mori, K. F. MacDorman, and N. Kageki, "The uncanny valley," IEEE Robotics and Automation Magazine, vol. 19, no. 2, pp. 98-100, 2012.

[5] H. Brenton, M. Gillies, D. Ballin et al., "The uncanny valley: does it exist," in Proceedings of Conference of Human Computer Interaction, Workshop on Human Animated Character Interaction, 2005.

[6] P. Ekman and W. V. Friesen, Manual for the Facial Action Coding System, Consulting Psychologists Press, 1978.

[7] P. Ekman and W. V. Friesen, Facial Action Coding System: Investigatoris Guide, Consulting Psychologists Press, 1978.

[8] J. Yan, Z. Wang, and S. Zheng, "Cognitive emotion research of humanoid expression robot," in Foundations and Practical Applications of Cognitive Systems and Information Processing, pp. 413-425, Springer, Berlin, Germany, 2014.

[9] F. De la Torre and F. Cohn J, "Facial expression analysis", in Visual Analysis of Humans, pp. 377-409, Springer, London, UK, 2011.

[10] S. Inversions, FaceGen modeller (Version 3.3) [computer software], Singular Inversions, Toronto, Canada, 2008.

[11] N. Endo, S. Momoki, M. Zecca et al., "Development of wholebody emotion expression humanoid robot," in Proceedings of the IEEE International Conference on Robotics and Automation (ICRA '08), pp. 2140-2145, Pasadena, Calif, USA, May 2008.

[12] K. Malchus, P. Stenneken, P. Jaecks, C. Meyer, O. Damm, and B. Wrede, "The role of emotional congruence in human-robot interaction," in Proceedings of the 8th ACM/IEEE International Conference on Human-Robot Interaction (HRI '13), pp. 191-192, IEEE, Tokyo, Japan, March 2013.

[13] R. C. Arkin and L. Moshkina, Affect in Human-Robot Interaction, Georgia Institute of Technology, Atlanta, Ga, USA, 2014.

[14] T. C. Wee, "Mechanical design and optimal control of humanoid robot (TPinokio)," The Journal of Engineering, vol. 1, no. 1, 2014.

[15] R. Zhu, J. Ren, Z. Chen et al., "Design and development of mechanical structure and control system for tracked trailing mobile robot," TELKOMNIKA Indonesian Journal of Electrical Engineering, vol. 11, no. 2, pp. 694-703, 2013.

[16] S. M. Sajadi, S. H. Mahdioun, and A. A. Ghavifekr, "Design of mechanical structure and tracking control system for $5 \mathrm{DOF}$ surgical robot," in Proceedings of the 21st Iranian Conference on Electrical Engineering (ICEE '13), pp. 1-6, Mashhad, Iran, May 2013. 

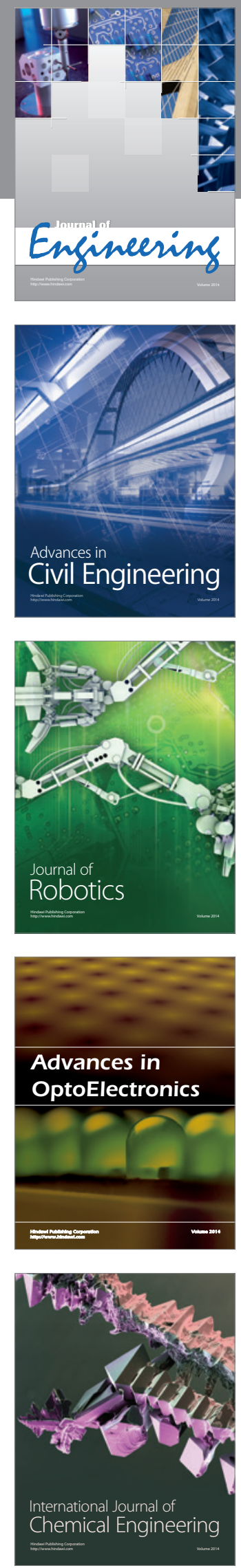

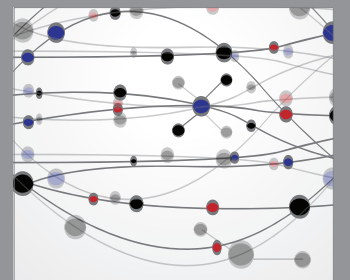

The Scientific World Journal
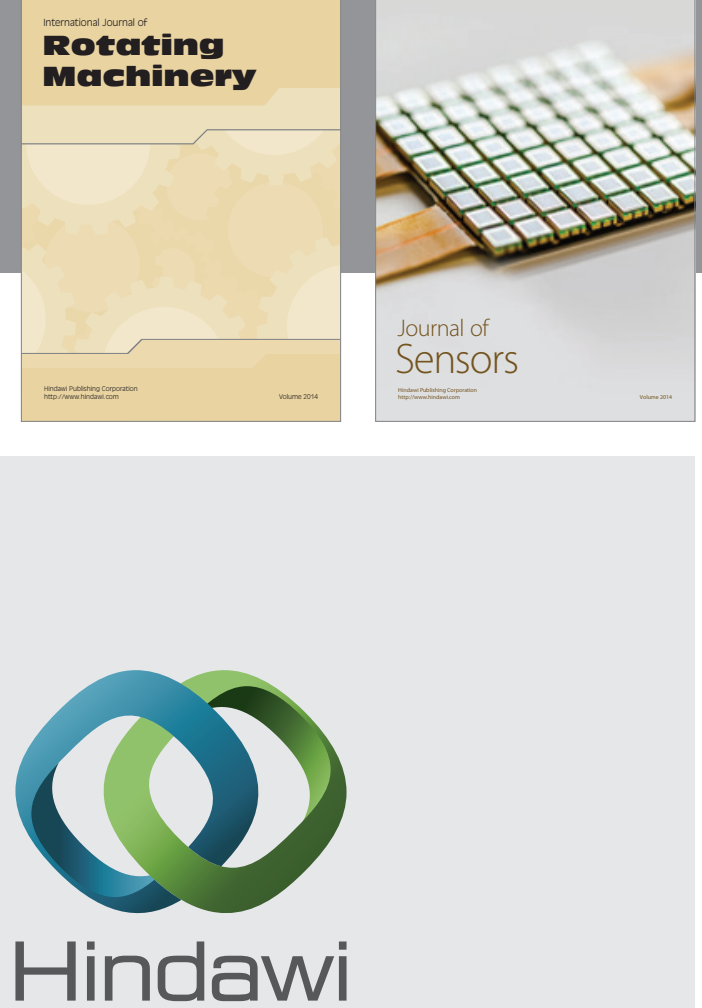

Submit your manuscripts at http://www.hindawi.com
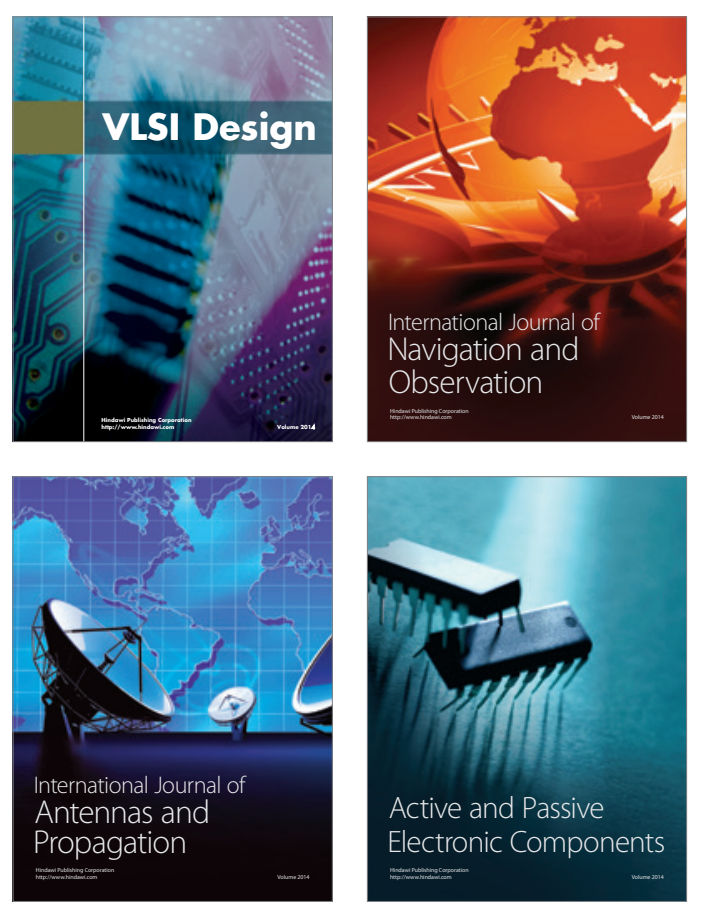
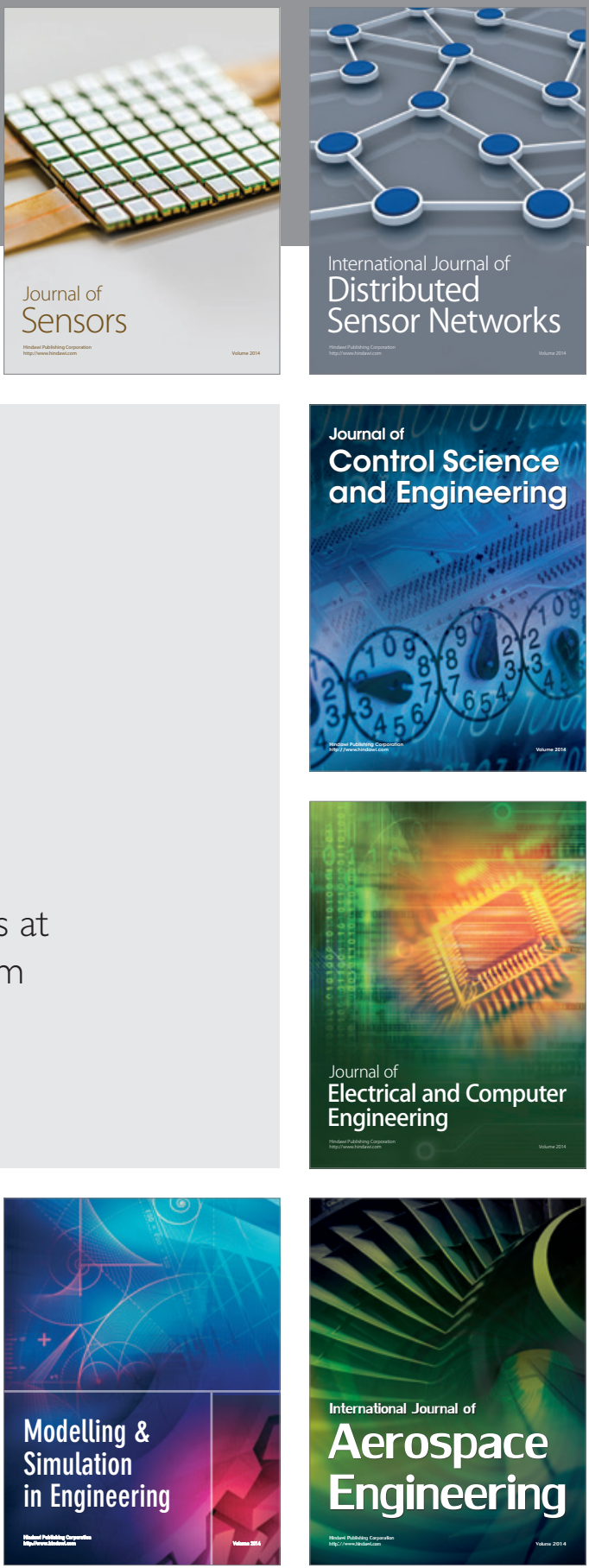

Journal of

Control Science

and Engineering
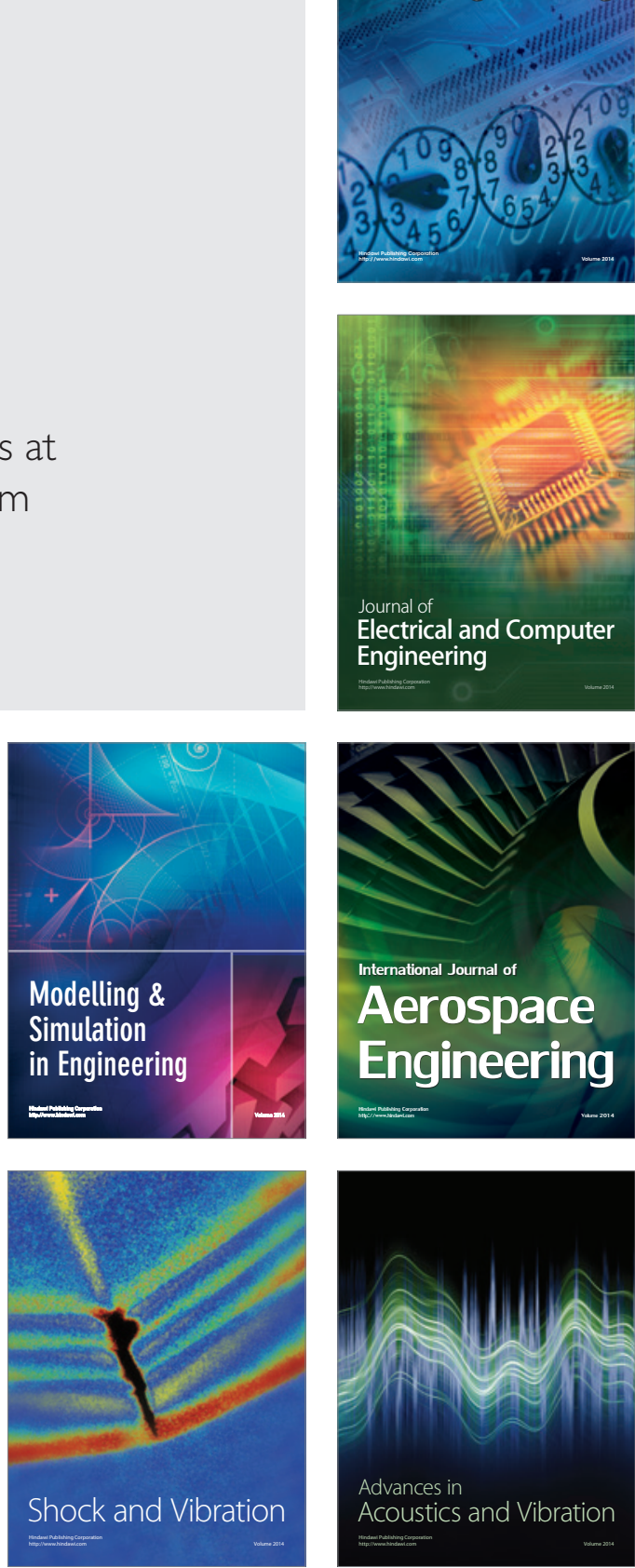